\title{
CUIDADOS DE ENFERMAGEM NO MANEJO DA DOR EM PACIENTES ADULTOS E IDOSOS EM CUIDADOS PALIATIVOS
}

\section{NURSING CARE IN PAIN MANAGEMENT IN ADULT AND ELDERLY PATIENTS IN PALLIATIVE CARE}

\section{ATENCIÓN DE ENFERMERÍA EN EL MANEJO DEL DOLOR EN PACIENTES ADULTOS Y DE ANCIANOS EN CUIDADOS PALIATIVOS}

\author{
Jéssica Luiza Ripani Rodrigues ${ }^{1}$, Silmar Maria da Silva², Isabel Yovana Quispe Mendoza², Ana Marília Cunha de Oliveira ${ }^{3}$
}

\section{RESUMO}

Objetivo: Identificar os cuidados de enfermagem no manejo da dor de pacientes adultos e idosos em cuidados paliativos. Método: Foi realizada uma revisão integrativa da literatura, de artigos nacionais e internacionais, que abordaram o tema "cuidados de enfermagem no manejo da dor de pacientes em cuidados paliativos", publicados no período de 2009 a 2019, em três bases de dados. Foram encontrados oito artigos que atenderam os critérios de inclusão e exclusão. Resultados: A partir da análise dos artigos, foram elaboradas duas categorias: a investigação da dor pela equipe de enfermagem em pacientes em cuidados paliativos e intervenções para o alívio da dor em pacientes em cuidados paliativos. Conclusão: Evidenciou-se a importância do vínculo entre o profissional e o paciente/família, no manejo da dor, além da assistência de forma holística. O pequeno número de estudos sobre o tema foi uma limitação para a pesquisa, ressaltando, assim, a necessidade de mais pesquisas nesta temática.

Descritores: Dor; Manejo da Dor; Conforto do Paciente; Cuidados paliativos na terminalidade da vida; Enfermagem.

\begin{abstract}
Objective: To identify nursing care in pain management of adult and elderly patients in palliative care. Method: This is an integrative literature review of national and international articles which addressed the "nursing care in pain management of patients under palliative care" published between 2009 to 2019 onto three databases. Eight papers that met the inclusion and exclusion criteria ere found. Results: From the analysis of the articles, two categories were elaborated: The investigation of pain by the nursing team in patients under palliative care; and Interventions for pain relief in patients under palliative care. Conclusion: Results evidenced the importance of the bond between the professional and the patient / family in the management of pain, as well as a holistic care. The small number of studies on the topic was a limitation for this study, thus emphasizing the need for more research on this topic.
\end{abstract}

Descriptors: Pain; Pain Management; Patient Comfort in end-of-life situations; Hospital Care; Nursing.

\section{RESUMEN}

Objetivo: Identificar los cuidados de enfermería en el manejo del dolor de pacientes adultos y ancianos en cuidados paliativos. Método: se realizó una revisión integral de la literatura, de artículos nacionales e internacionales, que abordó el tema "atención de enfermería en el manejo del dolor de pacientes en cuidados paliativos" publicado en el período de 2009 a 2019, en tres bases de datos. Se encontraron ocho artículos que cumplían los criterios de inclusión y exclusión. Resultado: A partir del análisis de los artículos, se elaboraron dos categorías: la investigación del dolor por parte del equipo de enfermería en pacientes en cuidados paliativos e intervenciones para el alivio del dolor en pacientes en cuidados paliativos. Conclusión: Se evidenció la importancia del vínculo entre el profesional y el paciente/familia en el manejo del dolor, además de la atención integral. El pequeño número de estudios sobre el tema fue una limitación para la investigación, enfatizando así la necesidad de más investigación sobre este tema. Descriptores: Dolor; Manejo del Dolor; Comodidad del Paciente; Cuidados Paliativos al Final de la Vida; Enfermería. 'Especialista em Saúde do Idoso, Prefeitura de Contagem, Minas Gerias, ${ }^{2}$ Doutora em Ciências, Universidade Federal de Minas Gerais, ${ }^{3}$ Mestranda em Gestão e Educação
em Saúde e Enfermagem., Universidade Federal de Minas Gerais.

Como citar este artigo:

Rodrigues JLR. Cuidados de enfermagem no manejo da dor de pacientes adultos e idosos em cuidados paliativos. Revista de Enfermagem do Centro Oeste Mineiro. 2020;10:e3680. [Access__]; Available in:__. DOI: http://doi.org/10.19175/recom.v10i0.3680 


\section{INTRODUÇÃO}

A Associação Internacional para o Estudo da Dor (IASP) define a dor como "uma experiência sensorial e emocional desagradável, associada a uma lesão efetiva ou potencial dos tecidos", que envolve também os aspectos psíquicos, espirituais e o ambiente ${ }^{(1)}$. A dor é singular a cada indivíduo, pois sofre interferência das memórias, experiências e emoções vivenciadas individualmente, afetando domínios importantes da experiência humana como os aspectos afetivos, cognitivos, comportamentais e socioculturais ${ }^{(2)}$. Destaca-se entre os sintomas geradores de sofrimento, pois é um sintoma de manejo complicado e de difícil avaliação, principalmente em pacientes idosos. $O$ paciente idoso, em fase avançada de uma doença pode apresentar características que dificultam a avaliação e o tratamento adequado ao controle da dor, como o comprometimento cognitivo funcional, comorbidades e condições de base, polifarmárcia e subnotificação(3).

Os Cuidados Paliativos (CP) são destinados a todos os pacientes que possuem doenças ameaçadoras da vida, havendo ou não a possibilidade de tratamento modificador da doença, tendo início no diagnóstico e finalizando no luto, incluindo a atenção a seus familiares ${ }^{(4)}$. Consiste em uma abordagem multiprofissional e interdisciplinar que tem como objetivo a melhoria da qualidade de vida do paciente e sua família, por meio da assistência holística, na prevenção e alívio do sofrimento por meio da identificação ágil, avaliação e tratamento da dor e dos demais sintomas desconfortantes, físicos, psicológico e espirituais.

Assim, para garantir o bem-estar e conforto do paciente em $\mathrm{CP}$, é primordial a atuação integrada de uma equipe multiprofissional, sendo que somente uma equipe, com múltiplas competências técnicas, pautada nos princípios do $\mathrm{CP}$, é capaz de proporcionar o conforto ao paciente $\mathrm{e}^{(5)}$.

A enfermagem é essencial à assistência paliativa, visto que possui como objeto de trabalho a prescrição de cuidados. $O$ enfermeiro deve estar atento às necessidades do paciente, não somente físicas, mas também psicológicas e espirituais. É importante, portanto, interpretar queixas verbais e não verbais, sobretudo, em se tratando do manejo da dor ${ }^{(6)}$.

Ressalta-se ainda o conhecimento da enfermagem, ao atuar no manejo e controle da dor, sintoma que está entre os maiores causadores de sofrimento e mais prevalente entre os pacientes em $\mathrm{CP}^{(7)}$. No entanto é desafiador, já que o conhecimento em CP ainda é deficiente à formação educacional dos profissionais, gerando consequências à qualidade da assistência ${ }^{(7)}$.

Diante do exposto, compreende-se que os cuidados de enfermagem, no manejo da dor em pacientes adultos e idosos em CP gera subsídios para uma assistência de melhor qualidade e eficácia, centrada no paciente. Assim sendo, o objetivo deste estudo foi identificar na literatura os cuidados de enfermagem voltados para o manejo da dor em pacientes adultos e idosos em cuidados paliativos.

\section{MÉTODO}

Foi realizada uma revisão integrativa da literatura, de artigos nacionais e internacionais que abordam o tema "cuidados de enfermagem no manejo da dor de pacientes em cuidados paliativos". Essa metodologia permite a integração de evidências na prática clínica, pela reunião e sintetização de evidências científicas, de forma organizada e sistemática, contribuindo para maior clareza e conhecimento do tema ${ }^{(8)}$.

A pesquisa foi elaborada, adotando as seis fases que estruturam a revisão integrativa: identificação do tema e elaboração de questão de pesquisa; definição dos critérios de inclusão e exclusão de estudos; extração de informações e categorização dos estudos; avaliação dos estudos incluídos na revisão integrativa; interpretação dos resultados; apresentação da revisão e síntese do conhecimento $^{(8)}$.

A questão norteadora da pesquisa foi: Quais são os cuidados de enfermagem no manejo da dor em pacientes adultos e idosos em cuidados paliativos? A busca aconteceu, no mês de setembro de 2019, utilizando-se as bases de dados do Scientific Electronic Library Online (SciELO), Biblioteca Virtual em Saúde (BVS) e PubMed. Foram incluídas no estudo as publicações científicas que respondiam à pergunta norteadora, ou seja, estudos primários que abordaram os cuidados da enfermagem no manejo da dor de pacientes adultos e idosos em CP, publicados entre os anos de 2009 a 2019, nos idiomas Português, Inglês e Espanhol.

Inicialmente ocorreu a análise dos títulos e resumos de todos os estudos identificados, dentro dos parâmetros estipulados. Além disso, foi realizada a leitura na íntegra daqueles estudos em que a análise do título e resumo foi insuficiente 
para a aplicação dos critérios de inclusão e exclusão.

Foram excluídas as publicações que não atenderam os critérios de inclusão, os estudos de revisão e demais tipos de estudo que não fossem artigo científico, além dos estudos duplicados. 0 processo da seleção dos estudos está exposto no fluxograma da Figura 1. A fim de ampliar a busca, realizou-se a busca reversa, na qual foram analisados os títulos do material referencial dos artigos selecionados.

Figura 1 - Fluxograma dos estudos selecionados da revisão integrativa. Belo Horizonte, MG, Brasil, 2019.

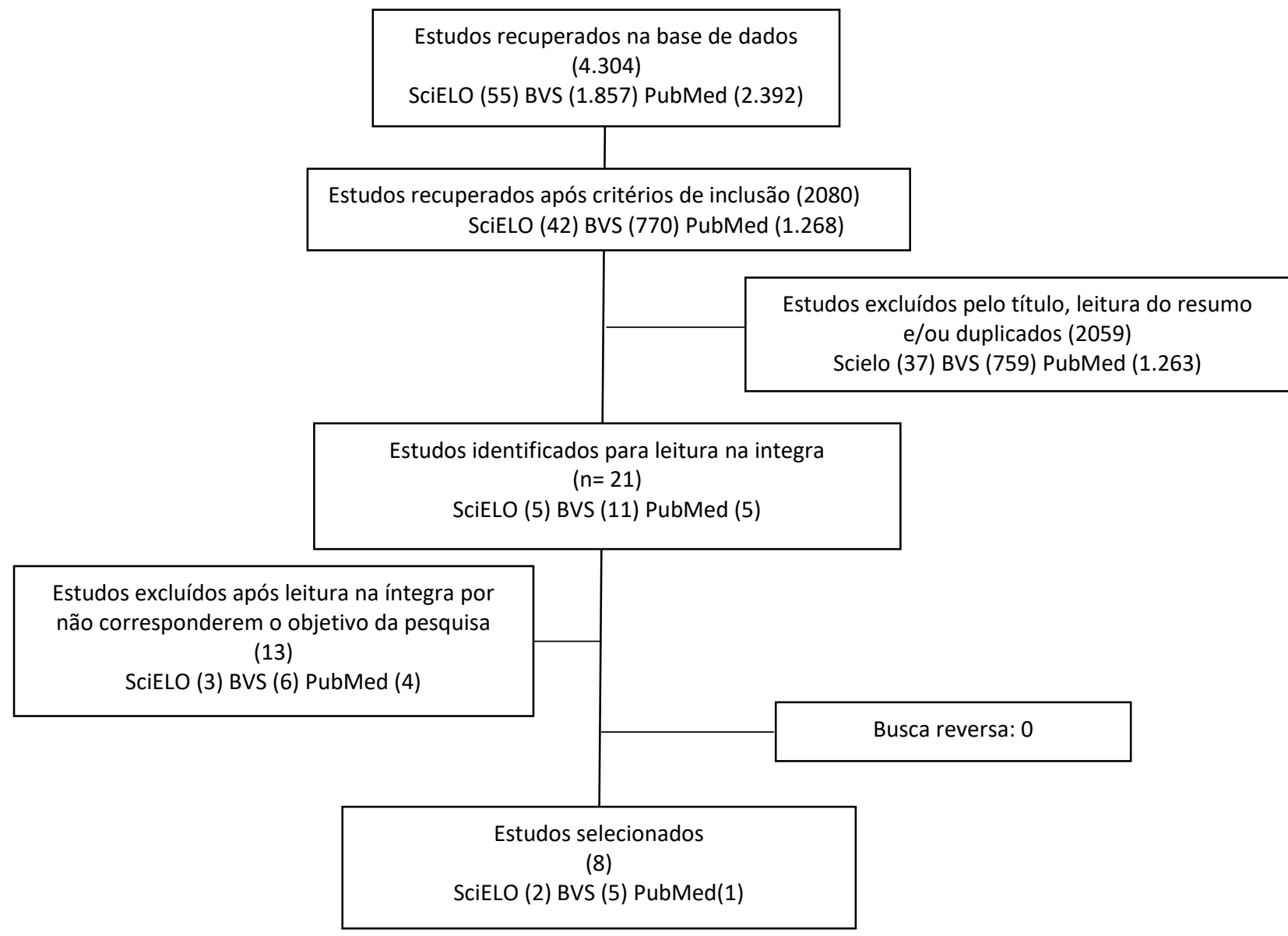

Fonte: Elaborada pelas autoras, 2019.

Para a extração de informações dos oito estudos selecionados foi elaborado um instrumento, para guiar a coleta de dados, que considerou as seguintes informações: título; base de dados onde foi detectada a publicação; autores; ano de publicação; país; idioma; local (País/Continente) onde foi realizado o estudo, qual o serviço de saúde onde ocorreu o estudo; profissionais participantes; objetivos do estudo; metodologia; principais resultados referentes aos cuidados de enfermagem no manejo dor e, por fim, as recomendações dos autores. Os estudos passaram por uma leitura ampla e remoção dos dados, guiada pelo instrumento elaborado. Optou-se por apresentar os resultados de forma descritiva, e para a análise dos dados, a categorização temática.

\section{RESULTADOS E DISCUSSÃO}

Dos oito estudos selecionados para essa revisão, identificou-se que os anos com mais publicações foram 2010 (2; 25\%) e 2018 (2; 25\%), e a maioria dos estudos - cinco $(62,5 \%)$ - foram identificados, na base de dados da BVS, com predomínio do idioma Inglês - seis (75\%) - seguida do Português, em dois (25\%) dos estudos. Não foram identificados estudos em Espanhol.

Quanto aos países de desenvolvimento dos estudos, quatro $(50 \%)$ se concentram no continente europeu, sendo três $(37,5 \%)$ do Reino Unido e um (12,5\%) originário da Suécia. Em segundo lugar, vem a América do Sul, com dois dos estudos (25\%) provenientes do Brasil. Há também estudos provenientes da Ásia e Oceania, 
conduzidos no Japão e na Austrália, respectivamente, sendo um estudo em cada país.

Os estudos aconteceram em três ambientes diferentes: Instituições de longa permanência (ILPI), hospitais e hospices. A metade dos estudos selecionados teve como local de estudo setores hospitalares específicos para CP, dois (25\%) em ILPI e hospices e dois (25\%) em hospitais gerais. As publicações que abordaram a investigação, identificação e avaliação da dor totalizaram seis (75\%) dos estudos. Destes, três estudos (50\%), além da identificação e avaliação, trouxeram também intervenções para o alívio da dor em pacientes.

A Figura 2 apresenta os principais resultados do manejo da dor e as recomendações dos autores.

Figura 2 - Descrição dos estudos incluídos na revisão integrativa, segundo principais resultados e recomendações dos autores. Belo Horizonte, MG, Brasil, 2019.

\begin{tabular}{|c|c|c|}
\hline Estudo & $\begin{array}{l}\text { Principais resultados quanto ao manejo } \\
\text { da dor }\end{array}$ & Recomendações dos autores \\
\hline Jansen BDW, et al./2018 ${ }^{(9)}$ & $\begin{array}{l}\text { Utilização da escala de dor Abbey para } \\
\text { avaliação da dor; } \\
\text { Demonstrou opiniões variadas a respeito } \\
\text { do uso da escala na avaliação da dor. }\end{array}$ & $\begin{array}{l}\text { Necessidade de maior apoio ao desenvolvimento } \\
\text { e aprimoramento de protocolos gerais e } \\
\text { abordagens de avaliação de dor existentes. }\end{array}$ \\
\hline $\begin{array}{l}\text { Skaczkowski G, et } \\
\text { al./2018(10) }\end{array}$ & $\begin{array}{l}\text { Banho de hidromassagem com } \\
\text { temperatura de acordo com a preferência } \\
\text { do paciente, resultou em melhora da dor, } \\
\text { ansiedade e bem-estar. }\end{array}$ & $\begin{array}{l}\text { São necessários maiores estudos acerca da } \\
\text { terapia à base de água. }\end{array}$ \\
\hline Jansen BDW, et al./2017(11) & $\begin{array}{l}\text { Pacientes não comunicantes precisam de } \\
\text { maior vigilância para identificação da dor; } \\
\text { Não há normas para a avaliação da dor na } \\
\text { instituição; } \\
\text { A avaliação da dor é centrada no paciente } \\
\text { por meio da observação comportamental; } \\
\text { Conhecimento holístico e vínculo são } \\
\text { facilitadores no manejo da dor. }\end{array}$ & $\begin{array}{l}\text { É essencial progredir no apoio, educação, } \\
\text { treinamento e aperfeiçoamento da equipe de } \\
\text { enfermagem para melhorar qualidade do } \\
\text { atendimento e conforto ao paciente. }\end{array}$ \\
\hline Brorson $\mathrm{H}$, et al./2014(12) & $\begin{array}{l}\text { Desvalorização da Enfermagem pode levar } \\
\text { ao subtratamento da dor; } \\
\text { Identificação da dor pela manifestação de } \\
\text { alterações de comportamento de sinais } \\
\text { individuais e singulares; } \\
\text { Boas práticas interprofissionais e } \\
\text { cooperação são importantes para alcançar } \\
\text { alívio da dor; } \\
\text { Os profissionais utilizam do manejo não } \\
\text { farmacológico para o alívio da dor, como: } \\
\text { toque, a proximidade (sentar-se ao lado), } \\
\text { segurar a mão, massagens, bolsas de água } \\
\text { morna, música preferida do paciente, } \\
\text { experiência gustativa. }\end{array}$ & $\begin{array}{l}\text { É preciso desenvolver uma ferramenta } \\
\text { padronizada de manejo da dor, para adoção na } \\
\text { prática clínica. }\end{array}$ \\
\hline Vargas MAO, et al./2013(13) & $\begin{array}{l}\text { Avaliação da dor é feita por } \\
\text { questionamento simples; } \\
\text { Conversa e a investigação de fatores } \\
\text { desencadeantes são ferramentas de } \\
\text { avaliação e manejo; } \\
\text { Exemplifica a mudança de decúbito com } \\
\text { uma ação de alívio da dor. }\end{array}$ & $\begin{array}{l}\text { Necessidade de locais especializados, bem } \\
\text { estruturados e com uma equipe qualificada para } \\
\text { atender o paciente em cuidados paliativos. }\end{array}$ \\
\hline $\begin{array}{l}\text { Yamamoto K, Nagata } \\
S / 2011^{(14)}\end{array}$ & $\begin{array}{l}\text { A técnica footbath, semelhante ao } \\
\text { escalda-pés, obteve resultado no alívio de } \\
\text { dor moderada. }\end{array}$ & $\begin{array}{l}\text { São necessários mais estudos acerca dos efeitos } \\
\text { benéficos da terapia. }\end{array}$ \\
\hline
\end{tabular}




\begin{tabular}{|c|c|c|}
\hline Estudo & $\begin{array}{l}\text { Principais resultados quanto ao manejo } \\
\text { da dor }\end{array}$ & Recomendações dos autores \\
\hline Soden K, et al./2010(15) & $\begin{array}{l}\text { O uso das escalas de avaliação da dor foi } \\
\text { relatado como demorado, e a relevância } \\
\text { das escalas foi questionada. } \\
\text { Enfermeiros possuem capacidade de } \\
\text { realizar avaliações holísticas, que } \\
\text { englobam o paciente integralmente. } \\
\text { As ações práticas de controle da dor } \\
\text { utilizadas foram: bolsas de calor, máquina } \\
\text { TENS (Estimulação Nervosa Transcutânea), } \\
\text { relaxamento: massagem, aromaterapia, } \\
\text { musicoterapia e alterações da } \\
\text { luminosidade. }\end{array}$ & $\begin{array}{l}\text { É necessário que os cursos de formação } \\
\text { profissional abordem a definição e avaliação da } \\
\text { dor aguda, formando assim profissionais } \\
\text { qualificados. } \\
\text { Para manejar a dor, é preciso uma equipe } \\
\text { multiprofissional. }\end{array}$ \\
\hline $\begin{array}{l}\text { Waterkemper R, Reibnitz } \\
\text { KS./2010(16) }\end{array}$ & $\begin{array}{l}\text { Compreensão do conceito dor total; } \\
\text { Não há instrumentos protocolados para a } \\
\text { avaliação da dor; } \\
\text { Avaliação da dor realizada de forma } \\
\text { individualizada e sistemática; } \\
\text { Diálogo, escuta ativa, vínculo e confiança } \\
\text { entre profissional e paciente/família são } \\
\text { importantes no manejo da dor. }\end{array}$ & $\begin{array}{l}\text { Necessidade de maiores estudos com foco no } \\
\text { manejo da dor pelos enfermeiros. }\end{array}$ \\
\hline
\end{tabular}

Fonte: Elaborada pelas autoras (2019).

No que tange às recomendações dos estudos selecionados verificou-se que três $(37,5 \%)$ indicaram a necessidade de maior número de pesquisas que busquem analisar o conhecimento dos profissionais de enfermagem e o gerenciamento da dor pela avaliação. Outros três artigos $(37,5 \%)$ recomendaram a necessidade de maior investimento na educação continuada, treinamentos, apoio na criação e implementação de protocolos do manejo da dor (nas instituições onde atuam?). Um estudo (12,5\%) apontou a carência de locais que estejam preparados e qualificados para atender pacientes com o perfil de controle álgico na fase final da vida. 0 desenvolvimento de uma ferramenta padronizada de avaliação e investigação da dor em pacientes que recebem CP foi recomendada por um dos estudos (12,5\%).

Após a análise dos artigos levantados, foram elaboradas duas categorias: Investigação da dor pela equipe de enfermagem em pacientes em $\mathrm{CP}$ e Intervenções para o alívio da dor em pacientes em CP.

\section{Investigação da dor de pacientes em cuidados paliativos}

Nos estudos em que o profissional de enfermagem relata como realiza a avaliação da dor (16-17), foi identificado o uso da primeira etapa do Processo de Enfermagem, isto é, a coleta de dados de enfermagem ou histórico de enfermagem ${ }^{(16-17)}$. Essa investigação é essencial para manejo e controle de sintomas, uma vez que, antes de planejar ou efetuar uma intervenção o enfermeiro deve ter a capacidade de avaliá-los ${ }^{(16-}$ 17). Nesse sentido, a identificação precoce de sintomas é essencial para que aconteça a prevenção e alívio do sofrimento, finalidade do cuidado paliativo.

Desta forma, objetiva-se um ambiente de cuidados totais interdisciplinares, ativos e integrais, que proporcionam a avaliação e, posteriormente, o tratamento adequado e eficaz para alívio da dor e demais sintomas ${ }^{(18)}$. Os resultados levantados evidenciaram a capacidade do enfermeiro para realizar avaliações holísticas, analisando a dimensão total da dor ao incluir não só o físico, mas também os aspectos psicológicos, espirituais e sociais do paciente, e de seus familiares ${ }^{(9,12,15)}$. A conduta mais citada para avaliação e identificação da dor foi a observação comportamental, uma vez que os locais de estudo referiram não possuir questionários e/ou escalas de avaliação protocolados ${ }^{(11,13,16)}$.

Observa-se que a dor interfere diretamente na qualidade de vida dos pacientes, que podem ter como uma das consequências o comportamento alterado $^{(9,11-12-13,15-16)}$. Os estudos ${ }^{(11-}$ ${ }^{12)}$ exemplificaram as mudanças comportamentais, como alterações de humor, podendo ir da irritabilidade, agressividade, agitação e gritos a depressão, desânimo e introversão, alterações no padrão sono-vigília, dificuldade na mobilidade e alterações de movimento de segmentos corporais, como utilizar a mão de forma diferente do habitual para se alimentar, e mudança do padrão da aceitação da dieta, como a inapetência ou apetite exagerado ${ }^{(11-12)}$. A avaliação da dor em 
pacientes não comunicantes foi considerada desafiadora. A estratégia utilizada pelos enfermeiros para a avaliação desse perfil de paciente não foi diferente dos demais. Novamente a observação comportamental de forma holística foi citada, com destaque à necessidade de vigilância da enfermagem nesses casos ${ }^{(11-12,15)}$.

A identificação das fácies de dor foi mencionada como algo complicado, de difícil diferenciação das demais situações desconfortantes, como o tédio e a fome ${ }^{(9,12,15)}$.De maneira perspicaz, os enfermeiros incluíram o familiar e/ou cuidador nesta diferenciação e identificação das fáceis, pelo vínculo mais estreito com o paciente $e$, consequentemente, por o conhecerem melhor. Assim, é primordial que a enfermagem inclua a família e o cuidador no processo de cuidar, para que se atinja uma assistência de qualidade, com vistas na prática colaborativa centrada no paciente $e^{(9,12,15)}$.

O uso da Escala de Dor Abbey (data), para avaliação de dor aguda ou crônica em idosos com demência avançada foi citado como uma ferramenta simples e insuficiente ${ }^{(9)}$. Constatou-se que para uma avaliação completa da dor, é necessário somar sua aplicação a outros fatores, como: o olhar holístico do enfermeiro e conhecimento atualizado do quadro clínico do paciente, bem como os sinais e sintomas recentes, histórico biopsicossocial, parecer de outros profissionais, documentos de prontuários e exames previamente realizados. Dessa forma, a complementação do uso desses instrumentos, aliado à avaliação da dor, baseado no processo de enfermagem, facilitarão a conduta e eficácia na terapêutica da dor ${ }^{(9)}$.

A Escala de Dor Abbey foi desenvolvida na Austrália e sua finalidade é identificação da dor em pacientes com demência severa. A escala avalia seis itens não verbais secundários a dor: vocalização, expressão facial, alteração na linguagem corporal, alteração comportamental, alterações fisiológicas (temperatura, ritmo cardíaco, transpiração), e alterações físicas, como lacerações na pele, artrite e lesões anteriores. Atribui-se um valor para cada item, mensurando a intensidade, sendo nota zero para ausência de dor, 1 para intensidade leve e 3 para severa. Feito isto, soma-se os valores atribuídos, que variam entre 0 e 18 pontos. 0 intervalo de 0 a 2, significa ausência da dor, entre 3 a 7 dor ligeira, 8 a 13 dor moderada e de 14 a 18 dor severa ${ }^{(19)}$.

O profissional enfermeiro por estar próximo do paciente no cumprimento de suas funções torna-se peça chave na avaliação da dor, especialmente aqueles com quadro de demência, por meio da observação das mudanças comportamentais e fisiológicas, como as citadas no parágrafo anterior. A busca diária de uma relação pautada no diálogo, associada à uma escuta de qualidade das questões trazidas pelo paciente e sua família é primordial para a criação desse vínculo enfermeiro-paciente e/ou enfermeiro-família ${ }^{(13,16)}$. É por meio do questionamento, da investigação dos fatores desencadeantes da dor, da observação e do contato diário com o paciente que o enfermeiro constrói uma relação de confiança e vínculo e a transforma em uma ferramenta de investigação desse sintoma ${ }^{(13,16)}$, constituindo-se a ponte que leva ao conhecimento dos padrões normativos do paciente $e^{(9,11-13,16)}$.

Um dos estudos identificados ${ }^{(12)}$ evidenciou a importância da relação interprofissional na avaliação da dor. Os enfermeiros relataram dificuldade de expor sua opinião clínica acerca do controle álgico do paciente, devido à falta de credibilidade, revelada pela fala e postura dos médicos ${ }^{(12)}$. Os enfermeiros acreditam que a dor dos pacientes é subtratada em razão desta fragilidade na relação interprofissional e, nesse sentido, ressaltam a necessidade de uma maior valorização da enfermagem ${ }^{(12)}$. No primeiro semestre de 2019 a Organização Mundial da Saúde (OMS) juntamente do Conselho Internacional de Enfermagem (CIE) lançaram a campanha intitulada Nursing Now (Enfermagem Agora) a qual tem como objetivo fortalecer os profissionais e dar maior destaque à profissão nos próximos dois anos ${ }^{(20)}$.

\section{Intervenções para o alívio da dor em pacientes em cuidados paliativos}

As intervenções de enfermagem são ações ou atividades que visam tratar um problema identificado e estão inclusas no PE, na fase de implementação. Estas ações são baseadas no conhecimento e julgamento clínico e têm como objetivo melhorar a qualidade de vida do paciente ${ }^{(17)}$. Nesse contexto, os enfermeiros utilizam tecnologias leves de cuidado para o manejo da dor dos pacientes adultos e idosos em $\mathrm{CP}^{(12-13,15)}$. Dentre as intervenções citadas pelos estudos para o manejo e controle da dor, e que estão dentro do grupo das tecnologias leves de cuidados podem ser elencadas: a conversa e o vínculo, o toque, segurar a mão, a proximidade, exemplificada como ato de estar ao lado do 
paciente, a musicoterapia e alterações ambientais, como aromaterapia e as mudanças da luminosidade $\mathrm{e}^{(12-13,15)}$.

O uso das tecnologias leves demonstram a capacidade do profissional do vínculo (de estabelecer vínculos?), bem como as habilidades de comunicação verbais e não verbais, que resultam em acolhimento e uma melhor assistência e resolutividade ${ }^{(21)}$. A comunicação é um componente indispensável na relação humana e é essencial no cuidado ${ }^{(4)}$. 0 indivíduo em cuidado paliativo possui vários conflitos, além da dor física, e deve ser entendido como um ser que está em sofrimento $^{(4)}$. Sendo assim, faz-se necessário permitir o compartilhamento de suas angústias, medos e anseios para que se sinta confortado, amparado e cuidado pelos profissionais de saúde ${ }^{(4)}$.

A conversa e a escuta qualificada comprovaram-se estratégias eficazes para conduzir o cuidado de forma humanizada àqueles que sofrem, auxiliando no controle álgico, vez que a comunicação eficiente constitui pilar para o surgimento do vínculo, pautado na confiança ${ }^{(22)}$, essencial no processo de investigação da dor.

A escuta atenta e de qualidade permite ao profissional que cuida de pacientes em CP identificar demandas reais e importantes para o paciente e que, se sanadas, poderão acarretar o conforto e o alívio de sofrimento ${ }^{(4)}$. A escuta terapêutica é um recurso ainda pouco utilizado pelos profissionais, seja por opção individual ou por desconhecimento. Na escuta terapêutica o sujeito verbaliza seus sentimentos, o que pode servir para subsidiar o cuidado de enfermagem ${ }^{(4)}$. $\mathrm{O}$ toque e a proximidade também são formas de comunicação, compreendidos na dimensão nãoverbal da comunicação. Ademais, o toque torna mais fácil a interação enfermeiro-paciente ${ }^{(4,23)}$.

Nas últimas décadas o toque vem sendo estudado e evidenciado como uma ação terapêutica ${ }^{(23-24)}$. Foi reconhecida a capacidade do contato físico de provocar alterações fisiológicas por meio do sensório, portanto, o toque é considerado um instrumento valioso na área de saúde ${ }^{(22-23)}$. Está comprovado cientificamente que as atitudes, como manter-se próximo, sentar-se ao lado, são intervenções eficazes na assistência emocional e espiritual de pacientes, pois, demonstram interesse e disponibilidade em ouvir e compreender ${ }^{(4)}$.

Um estudo brasileiro mencionou os benefícios em utilizar as Terapias Complementares nos $\mathrm{CP}^{(25)}$. Dentre os benefícios estão a promoção do relaxamento e a oportunidade de se criar um momento favorável na relação profissional-paciente. Esse estudo apontou que as terapias complementares auxiliaram na prevenção da depressão, trouxeram melhor qualidade de vida e potencializaram os efeitos dos analgésicos utilizados no controle da dor dos pacientes ${ }^{(25)}$.

A musicoterapia é uma terapia complementar de baixo custo, de fácil acesso e de alta aceitação pelos pacientes ${ }^{(25)}$. A música atua com um estímulo no enfrentamento daqueles que se encontram na terminalidade da vida, permitindo que os pacientes expressem emoções positivas. Além de que, ouvir música proporciona momentos prazerosos e relaxantes, restaura a paz e altera positivamente o humor ${ }^{(25)}$.

Duas técnicas mais elaboradas de assistência trouxeram resultados significativos na redução da dor em pacientes adultos e idosos em CP: o banho de hidromassagem e o footbath ${ }^{(10,14)}$. Estudo piloto australiano buscou compreender o potencial do banho de banheira de hidromassagem na percepção e redução da dor e ansiedade e aumento do bem-estar, em pacientes em fase final de vida. Constatou-se que banho de hidromassagem com temperatura variando de $10^{\circ} \mathrm{C}$ a $70^{\circ} \mathrm{C}$, respeitando a preferência do paciente e com duração de 20 a 30 minutos, resultou em melhora da dor, ansiedade e bem-estar ${ }^{(10)}$. A utilização da água como terapia é simples, promove o relaxamento e, indiretamente, reduz da ansiedade e a dor. Ficou entendido nesse estudo $^{(10)}$ que os fatores ansiedade, dor e bemestar estão associados e, portanto, não devem ser abordados separadamente. A utilização do banho de hidromassagem é de simples manejo para enfermagem, não necessitando de longos treinamentos, entretanto, a obtenção do equipamento adaptado para pacientes é de alto custo $^{(10)}$.

Outro estudo, realizado no Japão(14) abordou uma técnica nomeada footbath, que nada mais é que uma espécie de escalda-pés, onde se imerge os pés em água morna, envolvendo-os em um saco plástico e cobrindo-os com uma manta, após umectação dos membros com óleos. A intervenção engloba três fases: massagear as pernas com óleo por cinco minutos; mergulhar inicialmente as pontas dos dedos, e após a totalidade dos pés em uma bacia com água a $38^{\circ} \mathrm{C}$, sendo ajustada até $42^{\circ} \mathrm{C}$; envolver os membros inferiores com um saco plástico, incluindo a bacia até os joelhos e aplicar uma manta. Os pés ficam 
imersos por 20 minutos. Os pesquisadores comprovaram que a técnica do footbath proporciona aos pacientes: relaxamento, maior conforto, bem-estar e alívio da dor. Foi averiguado que houve uma diminuição da atividade do sistema nervoso simpático e redução do cortisol, o hormônio do estresse ${ }^{(14)}$. Esta, por sua vez já é uma técnica que demanda maior treinamento da equipe de enfermagem, mas por outro lado é mais acessível financeiramente, de fácil realização e pode ser praticada no leito do paciente.

\section{CONCLUSÃO}

Este estudo utilizou o método da revisão integrativa para identificar evidências disponíveis na literatura acerca o manejo da dor pela enfermagem em pacientes adultos e idosos em $C P$. Identificou-se que os trabalhos discutiram a contribuição da enfermagem na assistência no cuidado paliativo, apontando como principais resultados: o manejo da dor ${ }^{(10,13,14,15,16)}$, a

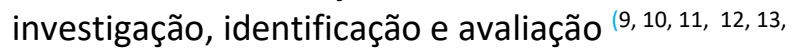
14) e as intervenções não farmacológicas para 0 alívio da dor ${ }^{(10,13,14,15,16)}$.

Quanto à assistência da enfermagem relacionada ao manejo da dor em pacientes, evidenciou-se maior atenção à investigação da dor por meio de sua identificação e avaliação. Compreendeu-se a importância do vínculo com o paciente e família, baseado na confiança, e obtida por entremeio de tecnologias leves de cuidado, no âmbito da comunicação verbal e não verbal no manejo da dor. A observação comportamental destacou-se como estratégia de avaliação e investigação da dor, e o conhecimento do estado normativo do paciente foi referido como fundamental para seu êxito.

Os resultados apresentados revelam a importância do cumprimento de todas as etapas do processo de enfermagem no cuidado da dor ao paciente em CP. Pois, a sua utilização possibilita à equipe de enfermagem organizar e direcionar suas ações às necessidades específicas dos pacientes, num contexto em que a pessoa é avaliada de forma integral.

Identificou-se, também, a capacidade do enfermeiro de manejar a dor de forma holística dando importância à relação profissional-paciente e sua família, quanto à sua atitude considerada bastante desafiadora na rotina de trabalho desse profissional. Ademais, constatou-se que no processo de cuidar o enfermeiro pode introduzir as Terapias Complementares e Integrativas, como a conversoterapia, a aromaterapia, o toque e mudanças ambientais, a musicoterapia, alterações na temperatura e luminosidade do ambiente. Estas são intervenções de cuidado indireto ao paciente, que proveem o bem-estar e o relaxamento e, consequentemente, a redução da dor e alívio de sofrimento de ordem física, psíquica e emocional.

O incipiente número de artigos disponíveis, a respeito dessa temática, foi uma limitação deste estudo. Portanto, apesar das intervenções reveladas serem de grande relevância à assistência de enfermagem do paciente com dor, no contexto do cuidado paliativo, ressalta-se uma carência de estudos primários e a importância do desenvolvimento de mais pesquisas que abordem esse tema.

\section{REFERÊNCIAS}

1- Merskey H, Fessard DA, Bonica JJ, Carmon A, Dubner R, Kerr FWL, et al. Termos de dor: Uma lista com definições e notas sobre $\mathrm{O}$ uso. Recomendado pelo subcomitê do IASP em taxonomia. Dor 1979 [cited 2019 Jun 9]; 6(3):24952. Available

in:

https://www.ncbi.nlm.nih.gov/pubmed/460932

2- Manfroi MN, Correia PMS, Franzoni WCC, Moraes LB, Stein F, Marinho A. Dor: O impulso na busca pela saúde por meio de práticas integrativas e complementares. BrJP 2019;2(4):316-20. DOI: 10.5935/2595-0118.20190058

3- Alexander K, Goldberg J, Korc-Grodzicki B. Cuidados paliativos e tratamento de sintomas em pacientes idosos com câncer. Clin Geriatr Med. 2016;32(1):45-62.

DOI: 10.1016/j.cger.2015.08.004

4- World Health Organization (WHO). Paliative care: Fact sheet $n^{\circ}$ 402. Geneva: WHO; 2015.

5- Araujo RL, Silva LA. Cuidados paliativos a comunicação como ferramenta no atendimento humanizado. Rev Augustus 2019 [citado em 12 mar 2020]; 24(48):169-81. Disponível em: http://revistas.unisuam.edu.br/ index.php/revistaaugustus/article/view/389

6- Franco HP, Stigar R, de Souza SJP, Burci LM. Papel da enfermagem na equipe de cuidados paliativos: A humanização no processo da morte e morrer. Gest Saúde 2017 [citado em 21 jul 2019]; 17(2)48-61. Disponível em: http://www.herrero.com.br/files/revista/file56fb 2faad065b8f7980ccdf2d0aa2da1.pdf

7- Costa ÁP, Poles K, Silva AE. Formação em cuidados paliativos: Experiência de alunos de medicina e enfermagem. Interface 
2016;20(59):1041-52. DOI: $\quad \underline{10.1590 / 1807-}$ 57622015.0774

8- Sousa LMM, Marques-Viera CMA, Severino SSP, Antunes AV. A metodologia de revisão integrativa da literatura em enfermagem. RIE 2017 [citado em 7 ago 2019]; 21:17-26. Disponível em: https://repositorio-

cientifico.essatla.pt/handle/20.500.12253/1311 9- Jansen BDW, Brazil K, Passmore $\mathrm{P}$, Buchanan $\mathrm{H}$, Maxwell D, Mcllfatrick S, et al. "A tool doesn't add anything". The importance of added value: Use of observational pain tools with patients with advanced dementia approaching the end of life-a qualitative study of physician and nurse experiences and perspectives. Int J Geriatr Psychiatry 2018;33(10):1346. DOI: 10.1002/gps.4931

10- Skaczkowski G, Moran J, Langridge J, Oataway $\mathrm{K}$, Wilson C. Effect of a spa bath on patient symptoms in an acute palliative care setting: $A$ pilot study. Complement Ther Clin Pract. 2018;32:100-2. DOI: 10.1016/i.ctcp.2018.05.004

11- Jansen BDW, Brazil K, Passmore P, Buchanan $H$, Maxwell D, Mcllfatrick SJ, et al. Exploring healthcare assistants' role and experience in pain assessment and management for people with advanced dementia towards the end of life: $A$ qualitative study. BMC Palliat Care 2017;16(1):6. DOI: 10.1186/s12904-017-0184-1

12- Brorson H, Plymoth H, Örmon K, Bolmsjö I. Pain relief at the end of life: Nurses' experiences regarding end-of-life pain relief in patients with dementia. Pain Management Nursing 2014;15(1):315-23.

DOI:

10.1016/j.pmn.2012.10.005

13- Vargas MAO, Vivan J, Vieira RW, Mancia JR, Ramos FRS, Ferrazzo $S$, et al. Ressignificando o cuidado em uma unidade especializada em cuidados paliativos: Uma realidade possível? Texto Contexto Enferm. 2013;22(3): 637-45. DOI: 10.1590/S0104-07072013000300009

14- Yamamoto K, Nagata S. Physiological and psychological evaluation of the wrapped warm footbath as a complementary nursing therapy to induce relaxation in hospitalized patients with incurable cancer: A pilot study. Cancer Nurs. 2011;34(3):185-92.

DOI:

10.1097/NCC.0b013e3181fe4d2d

15- Soden K, Ali S, Alloway L, Barclay D, Perkins P, Barker $S$. How do nurses assess and manage breakthrough pain in specialist palliative care inpatient units? A multicentre study. Palliat Med 2010;24(3):294-8.

DOI: $\underline{10.1177 / 0269216309355918}$
16- Waterkemper R, Reibnitz KS. Cuidados paliativos: A avaliação da dor na percepção de enfermeiras. Rev Gaúcha Enferm. 2010;31(1):8491. DOI: $10.1590 / \mathrm{S} 1983-14472010000100012$

17- Azevedo OA, Guedes ES, Araújo SAN, Maia MM, Cruz DALM. Documentation of the nursing process in public health institutions. Rev Esc Enferm USP 2019;53:1-8. DOI: 10.1590/s1980$\underline{220 \times 2018003703471}$

18- Almeida BP, Dias FSB, Cantú PM, Duran ECM, Carmona E. Atitude dos enfermeiros de um hospital público de ensino quanto ao processo de enfermagem. Rev Esc Enferm USP 2019;53:1-8. DOI: $10.1590 / \mathrm{s} 1980-220 \times 2018018203483$

19- Franco JRDM. Demência e dor: Reconhecimento e formas de abordagem [dissertação]. Lisboa: Universidade Lusófona de Humanidades e Tecnologias; 2018.

20- World Health Organization (WHO). Nursing now. Genebra: WHO; 2018 [cited 2019 Oct 25]. Available

in:

https://www.who.int/mediacentre/events/2018/ nursing-now/en/

21- Torres GMC, Figueiredo IDT, Cândido JAB, Morais APPA, Maria I. O uso de tecnologias leves no cuidado de pacientes hipertensos na Estratégia Saúde da Família. Esc Anna Nery 2018;22(3):1-8. DOI: 10.1590/2177-9465-ean-2017-0169

22- Silva RS, Oliveira ESA, Oliveira JF, Medeiros MOSF, Meira MV, Marinho CLA. Perspectiva do familiar/cuidador sobre a dor crônica no paciente em cuidados paliativos. Enfermería Actual de Costa Rica 2020;38:18-31. DOI: 10.15517/revenf.v0i38.37086

23- Ribeiro PCPSV, Marques RMD, Ribeiro MP. O cuidado geriátrico: Modos e formas de confortar. Rev Bras Enferm. 2017;70(4):830-7. DOI: 10.1590/0034-7167-2016-0636

24- O'Mathúna DP. Therapeutic touch for healing acute wounds. Cochrane Database Syst Rev. 2014; 29(7):CD002766.

DOI: 10.1002/14651858.CD002766.pub3

25- Schmid W, Rosland JH, von Hofacker S, Hunskår I, Bruvik F. Patient's and health care provider's perspectives on music therapy in palliative care: An integrative review. BMC Palliat Care 2018;17(1):32. DOI: 10.1186/s12904-018$\underline{0286-4}$

Nota: Este artigo fez parte do Trabalho de Conclusão de Residência Multiprofissional em Saúde do Idoso. Informamos que não houve agência de fomento.

Recebido em: $17 / 03 / 2020$

Aprovado em: 03/09/2020 
Endereço de correspondência:

Jéssica Luiza Ripani Rodrigues

Rua Setecentos e Setenta e Cinco, no 102, apartamento 301.

Bairro Diamante. CEP:30.644-158, Belo Horizonte, Minas

Gerais.

E-mail: jessica.ripani93@gmail.com 